\title{
Video-Assisted Pericardioscopy. How to Improve Diagnostic Efficacy in Pericardial Effusions
}

\author{
Paulo M. Pêgo-Fernandes, Fabio Fernandes, Barbara M. Ianni, Sandra Serson Rohr, \\ Isabela Miranda Bernardelli, Fabio B. Jatene, Sérgio A. Oliveira
}

São Paulo, SP - Brazil

\begin{abstract}
Objective - To assess, in a prospective way, the experience with video-assisted pericardioscopy obtained in patients with pericardial effusion of unclear etiology in the preoperative period.
\end{abstract}

Methods - From January 1998 to June 2000, 20 patients were operated upon with the aid of video-assisted pericardioscopy. On echocardiography, 17 of these patients had significant pericardial effusion, and 3 had moderate pericardial effusion. Video-assisted pericardioscopy was performed through a small incision of the Marfan type.

Results - The diagnosis of pericardial effusion was established as follows: idiopathic in $9(45 \%)$ patients, neoplastic in $4(20 \%)$, resulting from hypothyroidism in 3 (15\%), tuberculous in 2 (10\%), due to cholesterol in 1 (5\%), and chylopericardial in 1 (5\%). The biopsy was positive in $30 \%$ of the patients, and the etiology could not be defined in $45 \%$ of the patients.

Conclusion - Video-assisted pericardioscopy proved to be a method with low morbidity and a high index of diagnostic positivity. A high percentage of pericardial effusions are caused by viral infections, which are not diagnosed through current methods, being, therefore, classified as idiopathic.

Keywords: video-assisted thoracoscopy, biopsy, etiology, pericardial effusion

Instituto do Coração do Hospital das Clínicas - FMUSP

Mailing address: Paulo M. Pêgo-Fernandes - Rua Berlioz, 808 - 05467-000 - São Paulo, SP, Brazil - E-Mail: PauloPego@incor.usp.br

English version by Stela Maris C. e Gandour
Clinical investigation and the current complementary methods increase the frequency of the diagnosis of pericardial diseases; however, the same has not happened in regard to determining its etiology. This fact, many times, makes the treatment of patients difficult, especially in cases of recurring effusions. Therefore, new methods should be used. In an ideal situation, the method should confirm the etiological diagnosis, be therapeutical, and less invasive. From this perspective, video-assisted pericardioscopy is a relatively new procedure, which may be used with the above cited purposes.

The objective of this study was to assess our experience with video-assisted pericardioscopy, obtained in a prospective way, in patients with pericardial effusion of undefined etiology in the preoperative period, who were consecutively operated upon at the Heart Institute.

\section{Methods}

From January 1998 to June 2000, 20 patients with pericardial effusion were prospectively analyzed at the Heart Institute, School of Medicine University of São Paulo; these patients underwent video-assisted pericardioscopy in an attempt to diagnose more accurately the cause of pericardial effusion. Seven patients were of the male sex, 13 were of the female sex, and their ages ranged from 14 to 76 ( mean $=42.8 \pm 17$.6) years.

The signs and symptoms of this group of patients are shown in table I, fatigue and dyspnea being the major ones.

After clinical assessment, the type of involvement was characterized through echocardiography, and, when necessary, complemented with chest tomography and nuclear magnetic resonance. Based on echocardiographic assessment, 17 patients had significant pericardial effusion, and 3 had moderate pericardial effusion. Two patients (10\%) had signs and symptoms of restriction. Once the presence of effusion was confirmed and the conditions of the patients analyzed, a diagnostic procedure, video-assisted pericardioscopy, was proposed.

After sedation with general anesthesia, especially for 


\begin{tabular}{|lll|}
\hline \multicolumn{2}{|c|}{ Table I - Major signs and symptoms of the patients } \\
\hline & Patients & $\%$ \\
\hline Fatigue & 17 & 85 \\
Dyspnea & 17 & 85 \\
Thoracic pain & 6 & 30 \\
Anasarca & 3 & 15 \\
Ascites & 3 & 15 \\
Fever & 2 & 10 \\
\hline
\end{tabular}

those who were tenser and more obese, patients were put in the horizontal dorsal decubitus position, with a pad under the dorsum elevating the xiphoid process. We used only the peripheral venous access and routine monitoring with electrocardiography and oximetry.

Video-assisted pericardioscopy was performed through the classical Marfan incision ie, a small longitudinal median laparotomy of approximately $3-4 \mathrm{~cm}$ of length. After sectioning the linea alba, a longitudinal incision of the xiphoid process was performed (fig. $1 \mathrm{~A}$ and B). At that point, the first assistant surgeon, with the aid of retractors of the Richardson type, elevated the sternum so the surgeon could separate the 2 pleurae in the median line, through blunt dissection of these membranes. Then, the second assistant placed the instruments for video-assisted thoracoscopy close to the incision. With good visibility, the pericardium was incised and its fluid was withdrawn and referred for pertinent tests, such as cytology, biochemistry, and cultures (fig. 2). After the pericardial fluid was withdrawn, video-assisted pericardioscopy was performed, and included an inspection of the cardiac chambers and of the pericardium, in a search for implants both in the pericardium and in the epicardium (fig. 3). Pleural dissection was then completed. In the following step, a broad pericardial resection in the anterior portion of the heart was performed, aiming both at increasing the diagnostic opportunity, and at reducing the probability of recurrence. In the cases in which pericardial implants were found, a guided biopsy was performed and sent for anatomicopathological examination. The material was stained with hematoxylin and eosin, ZiehlNielsen, Grocott, and BH techniques and examined under optical microscope.

\section{Results}

The patients underwent video-assisted pericardioscopy, and drainage of the pericardial effusion revealed a serous fluid in 55\% of the cases. The characteristics of the pericardial fluid are listed in table II.

The anatomicopathological findings are listed in table III, unspecific chronic pericarditis being the most common.

Diagnostic positivity associated with video-assisted pericardioscopy was relatively high (30\%): 4 (20\%) neoplastic processes and $2(10 \%)$ cases of tuberculous pericarditis were diagnosed (tab. IV). The etiologic diagnosis was not obtained in $9(45 \%)$ patients.

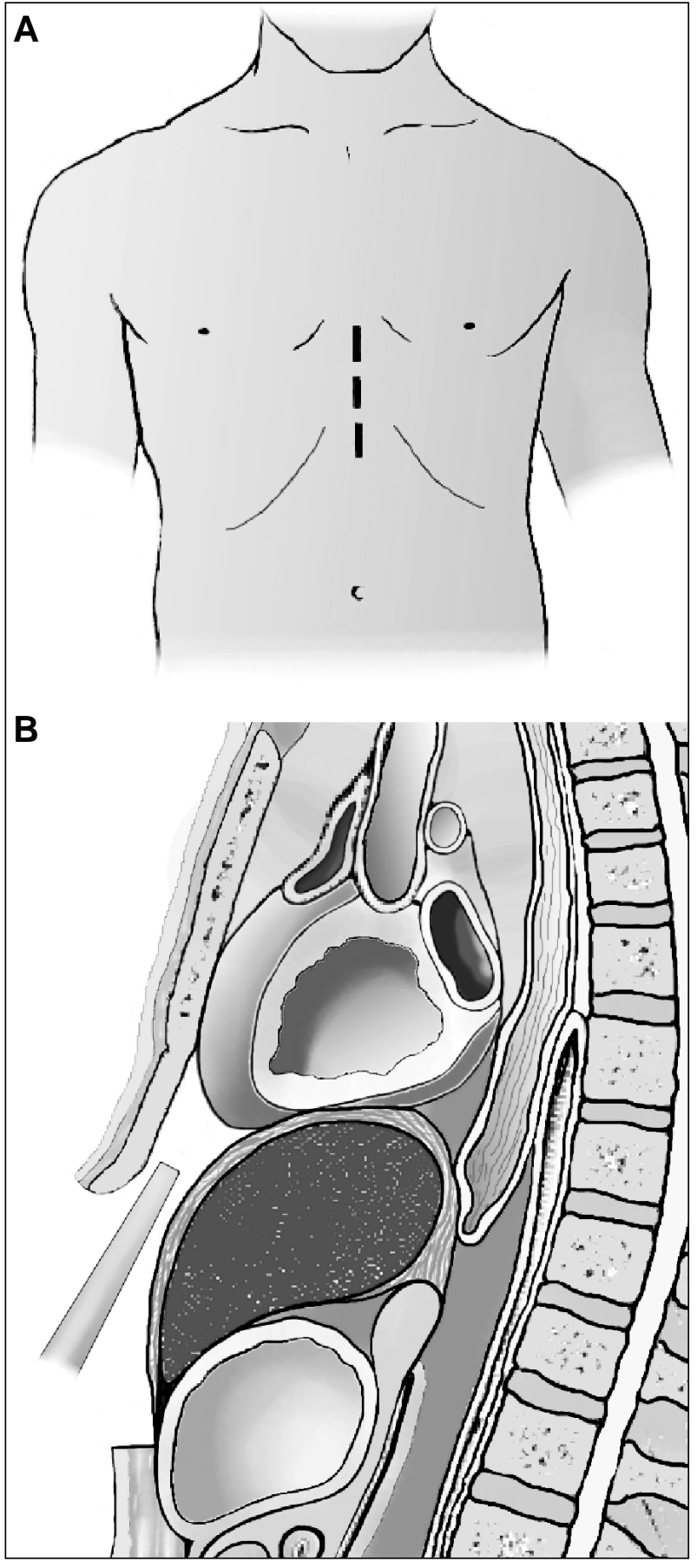

Fig. 1 - A) Incision of approximately 3-4 $\mathrm{cm}$ of length on the xiphoid process and linea alba; B) sketch of the entrance of the video equipment and visibility of the pericardium.

\section{Discussion}

Pericardial disease has several causes. Currently, noninvasive examinations allow an accurate diagnosis of pericardial effusions and constrictions; however, the etiologic diagnosis is not that easy to establish ${ }^{1}$. Invasive complementary methods are used for detecting the causes of pericarditis, mainly pericardial biopsy, which is a routine procedure in several centers ${ }^{2}$. The access route for pericardial 


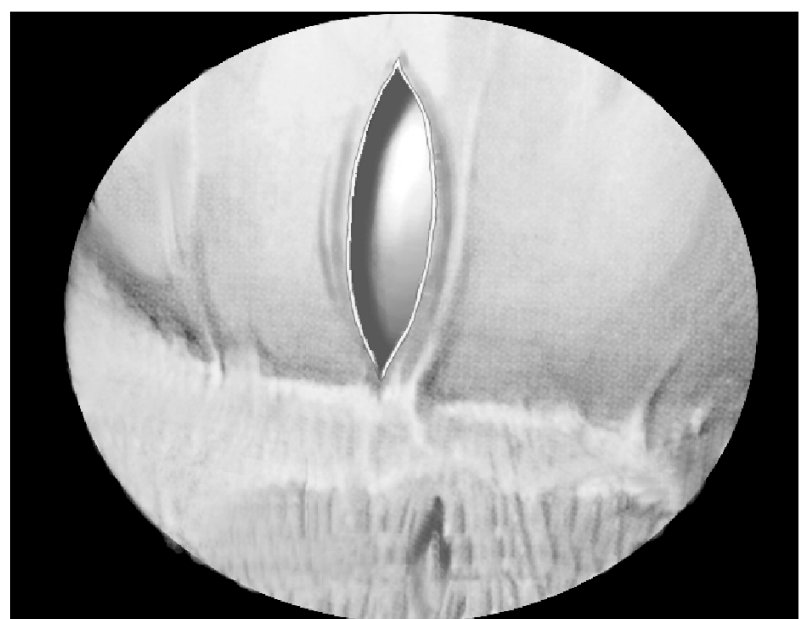

Fig. 2 - Visibility of the pericardium through the video equipment and beginning of the pericardial opening.

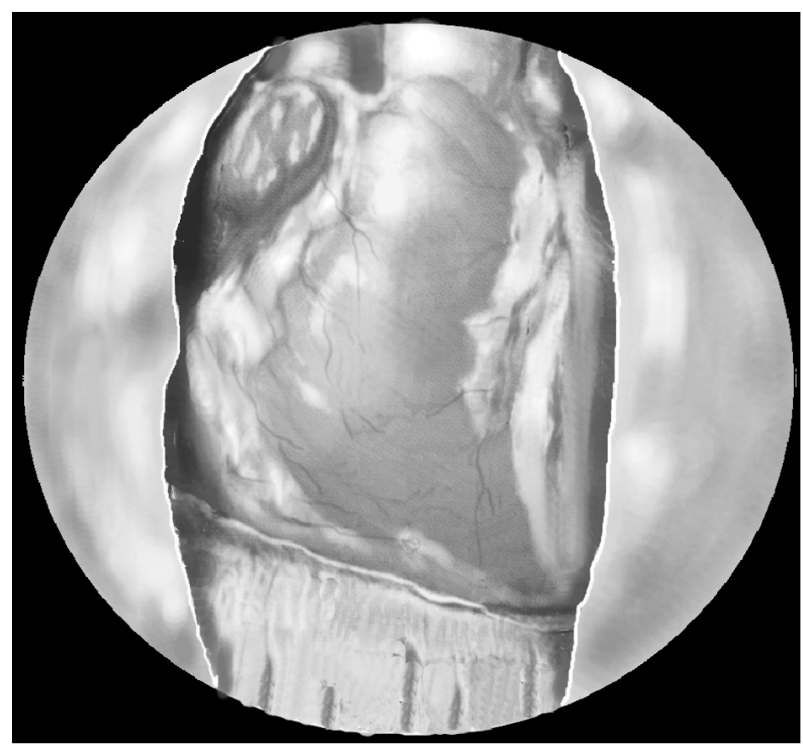

Fig. 3 - View of the heart after resection of the anterior part of the pericardium.

\begin{tabular}{|lll|}
\hline \multicolumn{3}{|c|}{ Table II - Characteristics of the fluid withdrawn } \\
\hline Fluid withdrawn & Patients & $\%$ \\
\hline Serous & 11 & 55 \\
Hemorrhagic & 8 & 40 \\
Chylous & 1 & 5 \\
\hline
\end{tabular}

biopsy may be transthoracic, median sternotomy, or subxiphoid ${ }^{3}$.

Identification of the cause of pericardial disease through the examination of the effusion is difficult, because cytological and microbiological limitations exist in the analysis of the pericardial fluid ${ }^{2}$. In regard to pericardial biopsy, the major limitation is the difficulty in obtaining pericardial tissue adequate for anatomicopathological study. This is due to the fact that the traditional subxiphoid approach allows very limited exposure of the pericardium,

\begin{tabular}{|lcc|}
\hline \multicolumn{2}{|c|}{ Table III - Anatomicopathological findings } \\
\hline Pericardium & Patients & $\%$ \\
\hline Unspecific chronic pericarditis & 7 & 5 \\
Neoplastic pericarditis & 4 & 20 \\
Tuberculous pericarditis & 2 & 10 \\
Others & 7 & 35 \\
\hline
\end{tabular}

\begin{tabular}{|lcc|}
\hline \multicolumn{3}{|c|}{ Table IV - Diagnosis obtained through video-assisted pericardioscopy } \\
\hline & Patients & $\%$ \\
\hline Neoplastic effusion & 4 & 20 \\
Effusion due to hypothyroidism & 3 & 15 \\
Tuberculous effusion & 2 & 10 \\
Effusion due to cholesterol & 1 & 5 \\
Chylopericardium & 1 & 5 \\
Idiopathic pericardial effusion & 9 & 45 \\
\hline
\end{tabular}

resulting in a low index of etiologic diagnosis and in rates of recurrence of pericardial effusion of up to $20 \%{ }^{4}$.

With the development of endoscopic equipment and refinement in surgical techniques, it was possible to develop a new technique, video-assisted pericardioscopy, that increases the sensitivity of the biopsy ${ }^{5,6}$.

Video-assisted pericardioscopy is a minimally invasive technique that allows excellent visibility of the pericardial sac; one may, under direct view, observe the most compromised pericardial regions, and, therefore, precisely select the site to be biopsied. Video-assisted pericardioscopy also allows the concomitant assessment of pleural and pulmonary affections when performed through a lateral approach ${ }^{7}$. Video-assisted thoracoscopy is associated with lower morbidity than that of thoracotomy ${ }^{7}$; in addition, few pulmonary dysfunctions are associated with this procedure ${ }^{8}$. In regard to the use of the subxiphoid via for video-assisted pericardioscopy, it has the advantage of not requiring drainage of the pleural cavity, reducing postoperative pain and avoiding pleural and pulmonary complications.

The usefulness of video-assisted pericardioscopy for diagnosing the cause of pericardial effusions is more evident when the results obtained with the traditional pericardial biopsy by the subxiphoid via are compared with those obtained when video-assisted thoracoscopic or video-assisted pericardioscopic techniques are used.

When studying the complementary methods for the etiologic diagnosis of pericardial effusion in 100 patients, Zayas et al. ${ }^{9}$ were able to define the etiology in $22 \%$ of the patients analyzed. The pericardial biopsy was negative in the 5 patients who underwent this procedure, and, therefore, could not contribute to clarifying the etiologic diagnosis.

Little et al. ${ }^{10}$, using the conventional technique of pericardioscopy, ie, without image amplification that is obtained in video-assisted pericardioscopy, in 17 patients who would undergo subxiphoid pericardiotomy for diagnosis or treatment of pericardial effusion, or both, obtained a positive result in $3(17.6 \%)$ patients. In 2 patients, a tumor could be 
seen and palpated on pericardiotomy, and, in the other patient, the tumor could be neither palpated nor seen, because it was in the posterior part of the pericardium. In the remaining $14(82.3 \%)$ patients, the results of pericardioscopy were negative, including the 4 patients who had positive cytological and microscopic examinations of the effusion and diffuse malignant infiltration of the pericardium. These 4 patients constituted a group of false-negatives: 2 had lung cancer and 2 had breast cancer.

Hui-Ping Liu et al. ${ }^{11}$ studied 28 patients with pericardial effusion, who had been previously diagnosed on echocardiography, and for whom video-assisted pericardioscopy had been indicated. Indications for this procedure were as follows: recurring effusion, pericardial effusion concomitant with pleural effusion, pericardial effusion concomitant with pulmonary or pleural mass, pericardial effusion following a surgical procedure, pericardial effusion after substernal esophagogastrectomy, and constrictive pericarditis in its initial phase. The objective of video-assisted pericardioscopy in the study by Hui-Ping Liu et al was to diagnose or treat pericardial effusion, or both. As a result, the authors concluded that the effusions were caused by: lung cancer in 11 patients, metastases of unknown primary neoplasia in 4 patients, esophageal cancer in 1 patient, uremia in 2 patients, cardiac procedures in 2 patients, tuberculosis in 6 patients, and chronic inflammation in 2 patients. After identifying the cause, the appropriate treatment was immediately started.

Initial experience of our colleagues with videopericardioscopy was reported by Barbosa et al $^{12}$ in 14 patients presenting positive diagnostic in $80 \%$ of the cases and compared with $30 \%$ of diagnostic obtained in the same patients through pericardiocentese and conventional biopsy.

In a study by Fernandes et al. ${ }^{2}$, with a case series of 38 consecutive patients at InCor, only $10.5 \%$ had the cause of the effusion defined with a biopsy through the subxiphoid via; in 2 patients the cause was tuberculosis, and, in 2 others, neoplasia. The remaining $(89.5 \%)$ had the diagnosis of unspecific chronic pericarditis. The authors concluded that the biopsy had not been an effective method for establishing the etiologic diagnosis of pericardial effusions, and suggested that the indication for biopsy should be individualized and restricted to those situations in which clinical data suggest effusion of neoplastic or tuberculous etiology.

These unsatisfactory results with the use of conventional biopsy obtained in our institution and in others, in addition to leading to the access of video-assisted thoracoscopic techniques, led to the use of video-assisted pericardioscopy. We used the same access route used in the traditional technique, ie, the subxiphoid via, because we believe that it is safer and has lower morbidity. When the effusion is small, greater effort is required for an appropriate visualization, because the natural space between the pericardium and the heart is small, making a more detailed inspection of the entire pericardium and epicardium difficult. In such cases, some authors suggest the injection of carbon dioxide. The lateral approach may also be chosen, through video-assisted thoracoscopy, and with this technique, selective bronchial intubation should be used. In cases in which the broadest possible resection of the pericardium is the objective more than determining the cause of the process, video-assisted thoracoscopy allows an easier visibility of the phrenic nerves, therefore, facilitating the resection.

In our case series, in which video-assisted pericardioscopy through the subxiphoid approach was performed in 20 patients, the diagnosis was idiopathic pericardial effusion in $9(45 \%)$ patients. Considering that the criteria for indication for the procedure and screening of patients was performed by the same clinicians and at the same institution, in which the rate of unspecific chronic pericarditis had been $89.5 \%{ }^{2}$, videoassisted pericardioscopy seems to be a huge step forward, even considering that the biopsy by itself provided the results in $30 \%$ of the patients, and the addition of the analysis of the fluid provided the diagnoses in $55 \%$. The authors believe that it is very difficult to exceed these values, because a significant percentage of pericardial effusions are caused by viral infections that are not diagnosed with the current methods; therefore, even using video-assisted pericardioscopy, pericardial effusion ends up being classified as idiopathic.

In conclusion, video-assisted pericardioscopy seems to be a technique with a high potential for diagnostic accuracy, and it should be indicated for patients with pericardial effusion, especially when neoplastic or tuberculous causes are suspected.

\section{References}

1. Corey GR, Campbell PT, van Trigt P, et al. Etiology of large pericardial effusions. Am J Med 1993; 95: 209-13.

2. FernandesF,IanniBM,ArteagaE,BenvenuttiL,MadyC. Valordabiópsiadepericárdio no diagnóstico etiológico das pericardiopatias. Arq Bras Cardiol 1998; 70: 393-5.

3. So T, Nakanishi R, Kohno H, Oe M, Yasumoto K. Video-assisted thoracoscopic pericardiectomy for severe pericardial effusions. J Cardiovasc Surg 1999; 40: $311-2$

4. Naunheim KS, Kesler KA, Fiore AC, et al. Pericardial drainage: subxiphoid vs transthoracic approach. Eur J Cardiothorac Surg 1991; 5: 99-104.

5. MackMJ, Aronoff RJ, Acuff TE, Douthit MB, Bowman RT, Ryan WH. Present role of thoracoscopy in the diagnosis and treatment of diseases of the chest. Ann Thorac Surg 1992; 54: 403-9.

6. Mack MJ, Landreneau RJ, Hazelrigg SR, Acuff TE. Video thoracoscopic management of benign and malignant pericardial effusions. Chest 1993; 103: 390S-3.
7. Nataf $P$, Cacoub P, Regan M, et al. Video-thoracoscopic pericardial window in the diagnosis and treatment of pericardial effusions. Am J Cardiol 1998; 82: 124-6.

8. Mack MJ. Thoracoscopic surgery for pericardial disease, 240-4, Chapter 29. In: Atlas of Video-Assisted Thoracic Surgery, ed. William T. Brown. W.B. Saunders Company 1994, Philadelphia, USA

9. Zayas R, Anguita M, Torres F, et al. Incidence of especific etiology and role of methods for especific etiologic diagnosis of primary acute pericarditis. Am J Cardiol 1995; 75: 378-82.

10. Little AG, Ferguson MK. Pericardioscopy as adjunt to pericardial window. Chest 1986; 89: 53-5.

11. Liu HP, Chang CH, Lin PJ, Hsieh HC, Chang JP, Hsieh MJ. Thoracoscopic management of effusive pericardial disease: indications and technique. Ann Thorac Surg 1994; 58: 1695-7.

12. Barbosa GV, Wender OCB, Souza JCP, et al. Pericardioscopia por via subxifoidea: sistematização e uso clínico. Arq Bras Cardiol 1991; 57(supl C): C62. 